\title{
Biomorphic Analog Devices based on Reaction-Diffusion Systems
}

\author{
Tetsuya ASAI and Yoshihito AMEMIYA \\ Department of Electronics, Hokkaido University \\ Kita 13, Nishi 8, Kita-Ku, Sapporo, 060-8628 Japan. \\ asai@sapiens-ei.eng.hokudai.ac.jp
}

\begin{abstract}
In this paper, we introduce new analog devices that eтиlate natural chemical systems, called the reaction-diffusion (RD) system. The RD system gives us a lot of clues and insights into developing new architectures based on a new paradigm in computing. We exhibit hardware RD devices and their typical operation, including the production of travelling and spiral waves in two-dimensional RD devices.
\end{abstract}

\section{Introduction}

Nonlinear oscillatory phenomena can be observed everywhere in the world. For instance, dissipative and autocatalytic reaction systems, which include almost every natural phenomenon, produce various spatiotemporal patterns through oscillatory reaction and the diffusion of chemical species. Chemical systems where the reaction and diffusion of chemical species coexist under a nonequilibrium condition are called reaction-diffusion (RD) systems [1]. Typical oscillatory behavior in RD systems can be observed in the Belousov-Zhabotinsky (BZ) reaction, which is a periodic oxidation-reduction phenomenon among liquid-state reagents. It produces a variety in rhythm and order in the form of propagating chemical waves [2].

The RD system gives us important clues into the relation between the chemical reaction and vital natural phenomena. Recent topics in this field concern the control of phase-lagged stable synchronous patterns in twodimensional (2D) space [3, 4], called modelock or spiral waves. Modelock is normally a negative factor in artificial systems because of the difficulty in controlling, and thus predicting, its dynamic behavior. It hampers the desired inphase synchronization of oscillator arrays, e.g., generating an irregular heartbeat or unpredictable skews in a 2D array of voltage-controlled oscillators for VLSI clocking. Several ideas for practical applications that use the properties of RD systems have also been proposed, e.g., ideas for chem- ical image processing [5, 6], optimal path planning [7], and binary logic processing [8]. These results suggest that natural systems that make actions primarily for themselves will help us to both understand RD systems and reconstruct them in artificial reaction media.

RD phenomena are usually observed in liquid-, gel- or gas-state media. Our interest was to construct an artificial $\mathrm{RD}$ system for a solid-state media, and to develop practical applications using the solid-state RD system that can cope easily with conventional digital computers. We developed this artificial RD system, which we call a reaction-diffusion chip, to imitate various natural RD phenomena (e.g., orders and rhythms, pattern formation, self organization in biological systems, etc.) on silicon VLSIs [9, 10, 11, 12].

Implementing RD systems in hardware (VLSI) has several advantages. Hardware RD systems are very useful for simulating RD phenomena, even if the phenomena never occur in nature. This implies that the hardware system is a possible candidate for developing an artificial RD system that is superior to the natural system. Moreover, hardware RD systems can operate at much faster speeds than actual RD systems. For instance, the velocity of chemical waves in a BZ reaction is $O\left(10^{-2}\right) \mathrm{m} / \mathrm{s}$ [13], while that of a hardware $\mathrm{RD}$ system will be over a million times faster than that of the BZ reaction, independent of system size $[9,10,11]$. This property is useful for developers of RD applications because every RD application benefits from high speed operations. These properties encouraged us to develop these new RD chips. In this paper, we introduce the hardware RD systems we have recently developed; i.e., i) a devicelevel implementation of a basic RD system and ii) an analog CMOS circuit that uses analog cellular-automaton to implement typical RD systems.

\section{The Reaction-Diffusion System}

Chemical reactions are formulated in terms of temporal differences in the concentration of chemical species. For example, if substance $x$ is dissolved in water, the temporal difference of the concentration of $x$ is expressed, by an 
ordinary differential equation (ODE), as

$$
\frac{d[x]}{d t}=-k[x]
$$

where $[x]$ represents the concentration and $k$ the rate constant. Although Eq. (1) is a linear ODE, most chemical reactions, including dissipative and autocatalytic reactions in natural systems, will be formulated by nonlinear ODEs with the right side of Eq. (1) represented by a polynomial of $[x]$. Nonlinear chemical reactions with multiple chemical species are thus represented by a set of nonlinear ODEs as

$$
\frac{d\left[x_{i}\right]}{d t}=f_{i}\left(\left[x_{1}\right],\left[x_{2}\right], \ldots,\left[x_{N}\right]\right), \quad(i=1,2, \ldots, N)
$$

where $N$ is the number of species and $f_{i}$ represents the nonlinear reactive functions that depend on several different reactive species $x_{i}$.

The Belousov-Zhabotinsky (BZ) reaction was formulated as Eq. (2). One well-known model of the BZ reaction is referred to as the two-variable Oregonator [1]. The dynamics are given by

$$
\begin{aligned}
& \frac{d\left[x_{1}\right]}{d t}=\frac{1}{\tau}\left(\left[x_{1}\right]\left(1-\left[x_{1}\right]\right)-a\left[x_{2}\right] \frac{\left[x_{1}\right]-b}{b+\left[x_{1}\right]}\right), \\
& \frac{d\left[x_{2}\right]}{d t}=\left[x_{1}\right]-\left[x_{2}\right],
\end{aligned}
$$

where $\left[x_{1}\right]$ and $\left[x_{2}\right]$ represent the concentration of $\mathrm{HBrO}_{2}$ and $\mathrm{Br}^{-}$ions, respectively, while $\tau, a$ and $b$ represent the reaction parameters. The value of $\tau$ is generally set at $\tau \ll$ 1 since the reaction rate of $\mathrm{HBrO}_{2}$ ion is much faster than that of $\mathrm{Br}^{-}$ions. The nullclines of the Oregonator where $d\left[x_{1}\right] / d t=0$ and $d\left[x_{2}\right] / d t=0$ are given by

$$
\begin{aligned}
& {\left[x_{2}\right]=\frac{\left[x_{1}\right]\left(\left[x_{1}\right]+b\right)\left(1-\left[x_{1}\right]\right)}{a\left(\left[x_{1}\right]-b\right)}, \quad\left(\equiv l_{1}\right)} \\
& {\left[x_{2}\right]=\left[x_{1}\right] . \quad\left(\equiv l_{2}\right)}
\end{aligned}
$$

A cross point of those two nullclines $\left(l_{1}\right.$ and $\left.l_{2}\right)$ represents the fixed point of the Oregonator.

Figure 1 shows the nullclines and trajectories of the Oregonator with typical parameter-values $\left(\tau=10^{-2}\right.$ and $b=0.02$ ). The value of parameter $a$ is set at 1 [Fig. 1(a)] and 3 [Fig. 1(b)]. Depending on the position of the fixed point, the Oregonator exhibits oscillatory or excitatory behavior. When $a=1$, the fixed point is located on nulllcline $l_{1}$ at which $d\left[x_{2}\right] / d\left[x_{1}\right]>0$. In this case, the Oregonator exhibits limit-cycle oscillations [Fig. 1(a)]. The oscillation represents periodic oxidation-reduction phenomena in the $\mathrm{BZ}$ reaction. On the other hand, the fixed point is located on nulllcline $i_{1}$ at which $d\left[x_{2}\right] / d\left[x_{1}\right]<0$ when $a=3$. Under this condition, the Oregonator exhibits excitatory behavior [Fig. 1(b)] and is stable at the fixed point as long as external stimulus is not given.

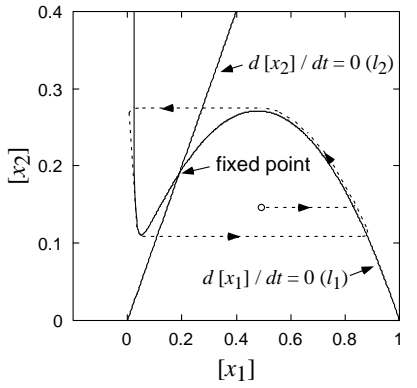

(a) limit-cycle oscillation

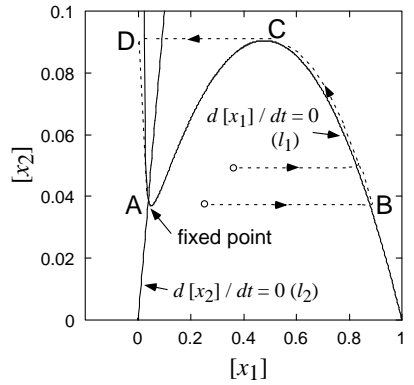

(b) excitation
Figure 1: Nullclines and trajectories of the Oregonator in (a) oscillatory mode and (b) excitatory mode.

In the Oregonator, three circulative states are introduced according to the oscillation phase; i.e., inactive (A), active $(\mathrm{B} \rightarrow \mathrm{C})$, and refractory periods $(\mathrm{D} \rightarrow \mathrm{A})$, as labelled in Fig. 1(b). The inactive, active, and refractory states represent a depletion in the $\mathrm{Br}^{-}$ion, an autocatalytic increase in the $\mathrm{HBrO}_{2}$ ion (oxidation of the catalyzer), and a depletion in the $\mathrm{Br}^{-}$ion (reduction of the catalyzer), respectively. When the Oregonator is inactive, it easily become active (A $\rightarrow$ B) by external stimuli. Then, it turns to the refractory state $(\mathrm{C} \rightarrow \mathrm{D})$. During the refractory state, the Oregonator can not be activated even if external stimuli is given.

It should be noted that Eqs. (1) to (4) represent the time difference of the chemical species at a point in the reaction space. If the spatial distribution of the chemical species is not uniform, the species will diffuse according to the gradient of the concentration of the species. Such a diffusivereaction system with multiple chemical species is referred to as a reaction-diffusion (RD) system, and is described by a set of partial differential equations as

$$
\frac{\partial\left[x_{i}\right](\mathbf{r}, t)}{\partial t}=D_{i} \nabla^{2}\left[x_{i}\right]+f_{i}\left(\left[x_{1}\right],\left[x_{2}\right], \ldots,\left[x_{N}\right]\right),
$$

where $\mathbf{r}$ represents the space, $\nabla^{2}$ the spatial Laplacian, $D_{i}$ the diffusion constant. A two-variable RD system on a 2D plane, which is referred to as a basic RD system, is described in terms of Eq. (7) as

$$
\begin{gathered}
\frac{\partial[u](x, y, t)}{\partial t}=D_{u}\left(\frac{\partial^{2}[u]}{\partial x^{2}}+\frac{\partial^{2}[u]}{\partial y^{2}}\right)+f_{u}([u],[v]), \\
\frac{\partial[v](x, y, t)}{\partial t}=D_{v}\left(\frac{\partial^{2}[v]}{\partial x^{2}}+\frac{\partial^{2}[v]}{\partial y^{2}}\right)+f_{v}([u],[v]),
\end{gathered}
$$

where $(x, y)$ represents the space, and $([u],[v])$ the concentration of two-different chemical species [1].

Figure 2 shows a schematic image of an alternative construction of the basic RD system. It consists of a 2D array of chemical oscillators; e.g., the Oregonators, where each oscillator is locally interconnected. An oscillator located at 


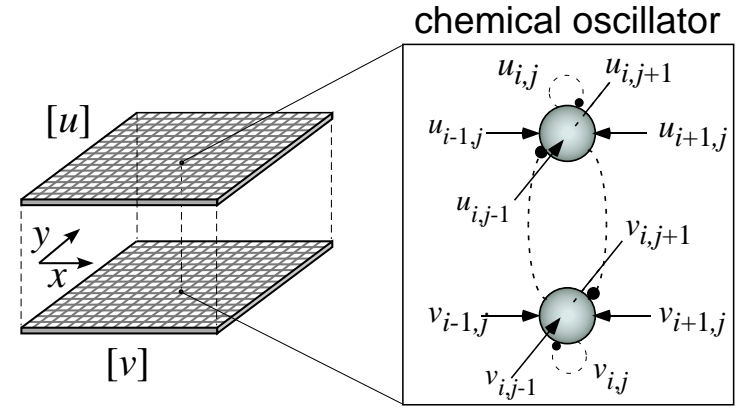

Figure 2: Discrete representation of basic RD system.

position $(i, j)$ has two system variables $\left[u_{i, j}\right]$ and $\left[v_{i, j}\right]$. The dynamics are defined as

$$
\begin{aligned}
& \frac{d\left[u_{i, j}\right]}{d t}=f_{u}\left(\left[u_{i, j}\right],\left[v_{i, j}\right]\right)+g_{i, j}^{u}, \\
& \frac{d\left[v_{i, j}\right]}{d t}=f_{v}\left(\left[u_{i, j}\right],\left[v_{i, j}\right]\right)+g_{i, j}^{v},
\end{aligned}
$$

where the functions $f_{u}$ and $f_{v}$ represent the nonlinear chemical interactions between $\left[u_{i, j}\right]$ and $\left[v_{i, j}\right]$, and where $g_{i, j}^{u}$ and $g_{i, j}^{v}$ represent the external inputs to the oscillator. External inputs are given to an oscillator so that activities $\left[u_{i, j}\right]$ and $\left[v_{i, j}\right]$ can diffuse in the $2 \mathrm{D}$ array of oscillators. Such inputs are given by five-point approximation of the Laplacian on the $2 \mathrm{D}$ rectangular grid as

$$
\begin{aligned}
g_{i, j}^{u} & =D_{u} \frac{[u]_{i-1, j}+[u]_{i+1, j}+[u]_{i, j-1}+[u]_{i, j+1}-4[u]_{i, j}}{h^{2}}, \\
g_{i, j}^{v} & =D_{v} \frac{[v]_{i-1, j}+[v]_{i+1, j}+[v]_{i, j-1}+[v]_{i, j+1}-4[v]_{i, j}}{h^{2}},
\end{aligned}
$$

where $h$ represents the distance between neighboring oscillators. The dynamics of the single oscillator [Eqs. (10) and (11)] thus represent the discrete expression of Eqs. (8) and (9). This expression allows us to understand the relation between natural RD systems and the physical hardware structure. Namely, 2D RD phenomena can be imitated on solid-state medium (e.g., VLSIs) where a lot of hardware oscillators are regularly arranged on the VLSIs with diffusive coupling among the local oscillator circuits.

Figure 3 shows numerical solutions to Eqs. (10) and (11) using an Oregonator with typical parameter-values. The nonlinear reactive functions $f_{u}(\cdot)$ and $f_{v}(\cdot)$ in Eqs. (10) and (11) are replaced with the right side of Eqs. (3) and (4), respectively, with the transformation of system variables $\left(\left[x_{1}\right] \rightarrow\left[u_{i, j}\right]\right.$ and $\left.\left[x_{2}\right] \rightarrow\left[v_{i, j}\right]\right)$. Each oscillator was set at excitatory mode $(a=3)$, and the values of the rest of the parameters were $h=0.01, D_{u}=5 \times 10^{-4}$, $D_{v}=0, \tau=10^{-2}$ and $b=0.02$. The solution was numerically obtained by solving the ODEs using the fourth-order

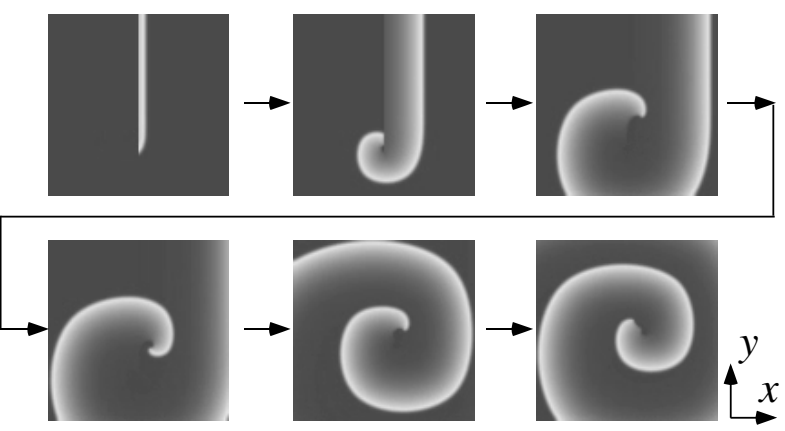

Figure 3: Typical numerical results of a RD system using the Oregonator.

Runge-Kutta method. At each side of the square reactionspace, we applied the Neumann boundary condition:

$$
\nabla[u]=\nabla[v]=(0,0),
$$

where $\nabla=(\partial / \partial x, \partial / \partial y)$. For example, the values of $\left[u_{0, j}\right]$ and $\left[u_{N+1, j}\right]$ are treated as those of $\left[u_{1, j}\right]$ and $\left[u_{N, j}\right]$, respectively. In Fig. 3, the values of $v_{i, j}$ are represented in grayscale $\left(v_{i, j}=0\right.$ : black, $v_{i, j}=1$ : white). Several oscillators adjacent to the inactive oscillators were initially set at a refractory state (left side of the white bar in Fig. 3). The inactive oscillators adjacent to the white bar were suppressed by the adjacent oscillators in the refractory state (oscillators in the white bar). The inactive oscillators then entered an active, inactive, or refractory state, depending on the degree of the refractory condition. When the inactive oscillators were in an active or inactive state, the tip of the bar rotated inward, resulting in the generation of the spiral patterns that are typically observed in a $\mathrm{BZ}$ reaction.

\section{A Reaction-Diffusion Device using Minor- ity Carrier Transport}

The RD device we proposed is illustrated in Fig. 4. It consists of four-layer $p$ - $n$ - $p$ - $n$ diodes arranged regularly on a silicon substrate. Each $p-n-p-n$ diode is connected with a capacitor and a current source to form a relaxation oscillator, as shown in Fig. 5. This oscillator acts as a unit cell that imitates a chemical reaction, a substrate-depleted reaction. Therefore, we call the oscillator a reaction cell. The reaction cell produces minority carriers (electrons) in the inner $p$ region during the oscillation cycle. Our idea is to use the minority carriers as diffusion substances; i.e., electrons produced by a reaction cell will travel through the inner $p$ region by diffusion and reach the neighboring reaction cells to activate these cells (see Fig. 4). A two-dimensional RD system can thus be constructed on a silicon chip.

The state of the reaction cell can be represented by two variables, i.e., charge $u$ stored on the capacitor and charge $v$ 


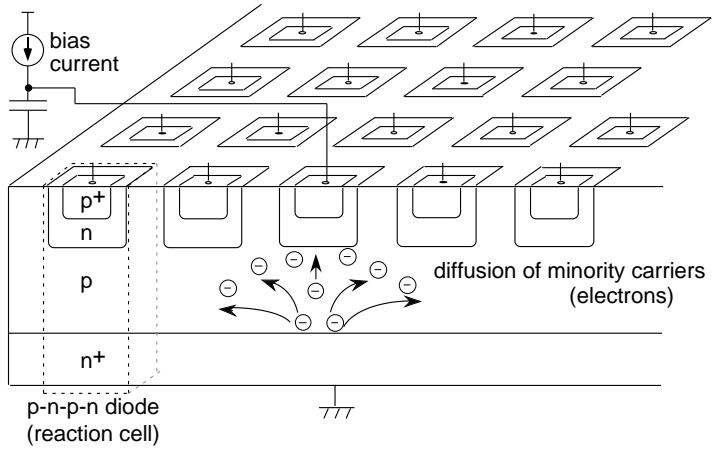

Figure 4: The reaction-diffusion device (RD device) consisting of an array of $p-n-p-n$ diodes.

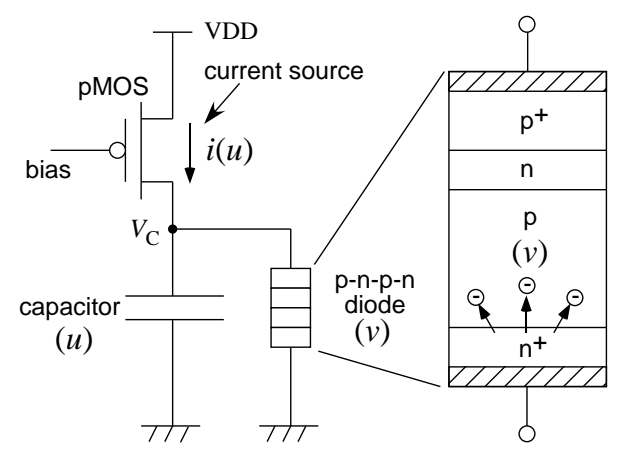

Figure 5: The reaction cell consisting of a $p-n-p-n$ diode, a capacitor, and a current source. The current source consists of a $p$ MOS transistor.

of the minority carriers in the $p-n-p-n$ diode. The dynamics of the reaction cell are described by

$$
\begin{aligned}
& \frac{d u}{d t}=i(u)-\frac{u}{\tau(u, v)} \\
& \frac{d v}{d t}=-v+\frac{u}{\tau(u, v)}
\end{aligned}
$$

where the charges $u$ and $v$ are normalized. The bias current $i(u)$ from the $p$ MOS current source is a function of $u$ and is also normalized. The characteristic of the $p-n-p-n$ diode is represented by nonlinear function $\tau(u, v)$. Minority-carrier charge $v$ increases through a multiplication process caused by the feedback mechanism of the $p-n-p-n$ diode, while capacitor charge $u$ decreases by an amount equal to the increased minority carriers. The operation is categorized as a substrate-depleted reaction.

The reaction cell can be oscillatory (astable) or excitatory (monostable) depending on the supply voltage, VDD. It is oscillatory if the VDD is higher than the breakover voltage $V_{\mathrm{B}}$ of the $p-n-p-n$ diode, and excitatory if the VDD is lower than the $V_{\mathrm{B}}$. In the oscillatory condition (VDD $>V_{\mathrm{B}}$ ), the capacitor is charged by bias current $i(u)$. As a

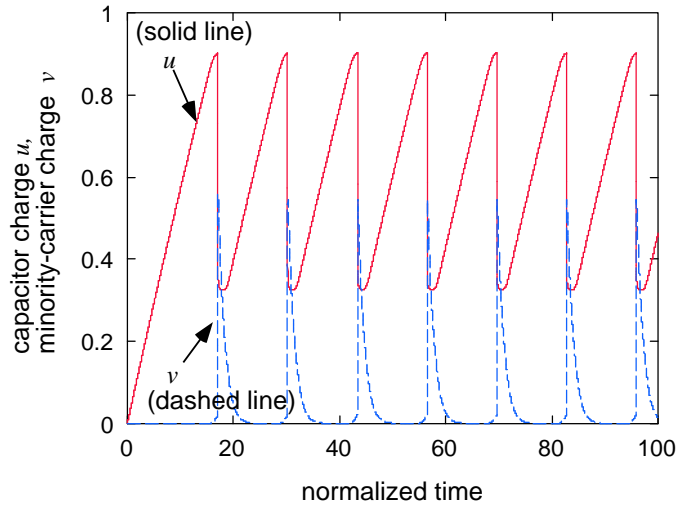

Figure 6: Dynamical properties of the oscillatory reaction cell (simulation). (a) Time evolution; (b) limit-cycle attractor on the $u-v$ plane.

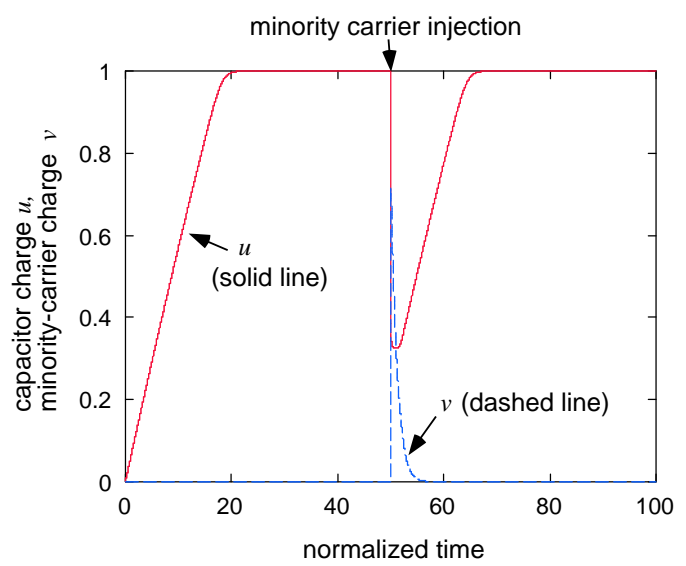

Figure 7: Time evolution in the excitatory reaction cell (simulation).

result, the capacitor charge $u$ increases until the capacitor voltage $V_{\mathrm{C}}$ reaches the breakover voltage $V_{\mathrm{B}}$. At this point, the breakover of the $p-n-p-n$ diode starts and the minority carriers are injected from the $n+$ region to the $p$ region. The autocatalytic multiplication of the minority carriers then occurs to turn the diode on. The stored charge on the capacitor flows into the diode, making the capacitor charge $u$ (therefore capacitor voltage $V_{\mathrm{C}}$ ) decrease. Consequently, the diode is turned off. The reaction cell repeats this cycle and produces the oscillatory dynamics. Figure 6 illustrates an example of the numerical solutions to Eqs. (13) and (14). Figure 6(a) shows the relaxation oscillations for variables $u$ and $v$. The limit-cycle attractor is shown in Fig. 6(b).

In the excitatory condition (VDD $<V_{\mathrm{B}}$ ), the capacitor voltage $V_{\mathrm{C}}$ cannot reach the breakover voltage $V_{\mathrm{B}}$ because $V_{\mathrm{C}}$ does not exceed the supply voltage VDD (bias current $i(u)$ becomes 0 when $V_{\mathrm{C}}$ increases up to VDD). In this con- 

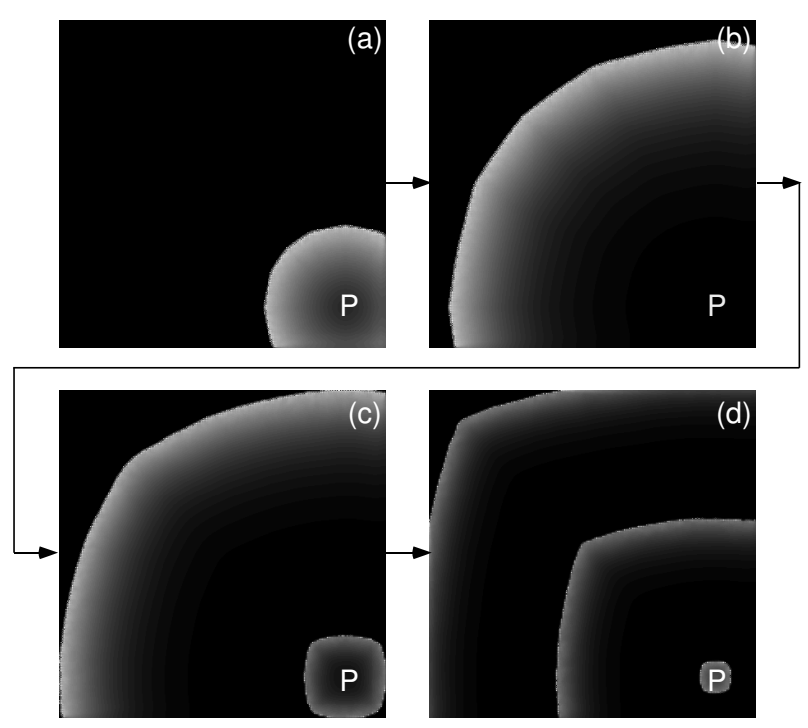

Figure 8: Generation of spreading concentric patterns in a RD device (simulation).

dition, the $p-n-p-n$ diode turns on only when the minority carriers are injected from the neighboring diodes. Figure 7 shows an example of the excitatory behavior of the reaction cell. The cell settles down in the stable state of $u=1$ and $v=0$, and no further change occurs as long as the minority carriers are not injected. In the simulation, minority carriers were injected from the outside when time $=50$. The $p-n-p$ $n$ diode turned on once by this injection, and then returned to the stable state,

We designed a 2D RD device by arranging the reaction cells on a plane and confirmed the device operation by computer simulation. We used the following RD equations that describe spatiotemporal dynamics at the position of each reaction cell:

$$
\begin{aligned}
& \frac{\partial u(x, y)}{\partial t}=i(u)-\frac{u}{\tau(u, v)} \\
& \frac{\partial v(x, y)}{\partial t}=D_{v} \nabla^{2} v-v+\frac{u}{\tau(u, v)} .
\end{aligned}
$$

where $(x, y)$ are the rectangular coordinates of any point on the RD-device plane, and $D_{v}$ is the normalized diffusion coefficient. In other positions, where no reaction cell exists, we used the following equations:

$$
\frac{\partial u(x, y)}{\partial t}=0, \quad \frac{\partial v(x, y)}{\partial t}=D_{v} \nabla^{2} v-v
$$

We solved these equations numerically using the fourthorder Runge-Kutta method.

Figure 8 shows a result for a device with $200 \times 200$ excitatory reaction cells. The spatial density of the minority carriers is represented in grayscale ( $v=0$ : black, $v=1$ : white).
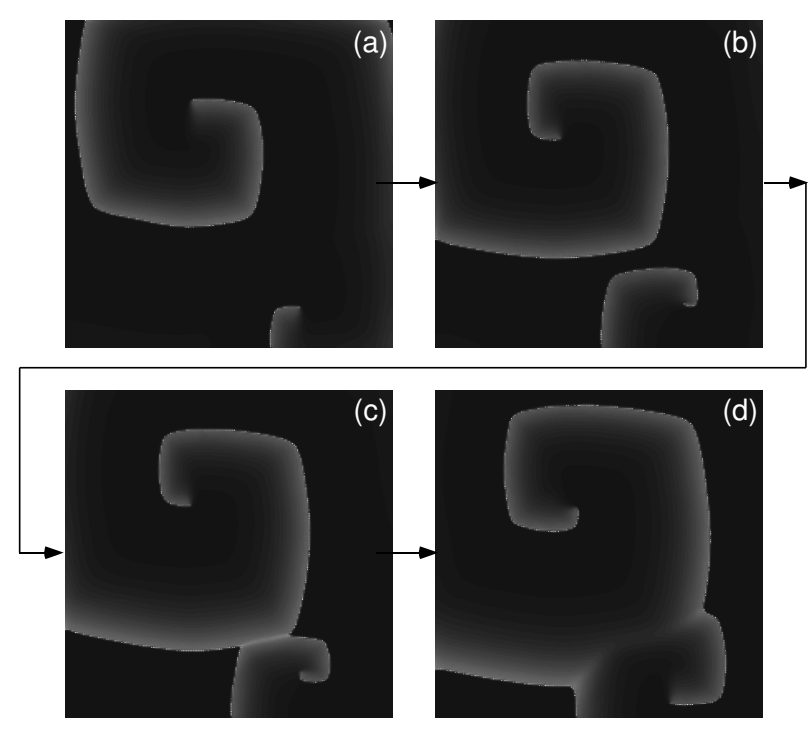

Figure 9: Generation of rotating spiral patterns in a RD device (simulation).

With periodic injection of minority carriers at a point (e.g., $P$ in Fig. 5), the RD device produced spreading concentric waves of the minority carriers. This result indicates that the injected carriers diffused around the injection point and successfully induced a chain of reactions in the cells.

Figure 9 shows the result of the excitatory RD device without any external injection of minority carriers. With an appropriate initial pattern of minority-carrier densities, the RD device produced rotating spiral patterns of the minority carriers. Notice that the wave disappears at collision points [Figs. 9(c) through (d)] because of the depletion of the minority carriers. This is the same phenomenon as observed in natural RD systems.

\section{Modelling the Reaction-Diffusion System with Analog Cellular-Automaton}

A cellular-automaton (CA) system is a discrete dynamical system whose behavior is completely specified in terms of finite local interactions $[14,15,16]$, and is thus suitable for VLSI implementation $[17,18]$. The discrete expression of basic RD models, introduced in $\S 2$, has a lot of similarity to the CA system. First, it consists of a number of identical cells (processors) and local connections among these cells. Second, these cells are regularly arrayed on a $2 \mathrm{D}$ rectangular grid. Thus, the basic RD model can be replaced with a CA model by assuming that: i) each cell represents interactions between species $u_{i, j}$ and $v_{i, j}$ at a specific point $(i, j)$, ii) concentration of chemical species $\left(\left[u_{i, j}\right],\left[v_{i, j}\right]\right)$ is represented by values of system variables in each cell, and iii) 


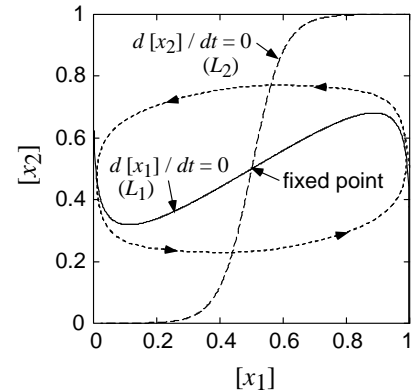

(a) limit-cycle oscillation

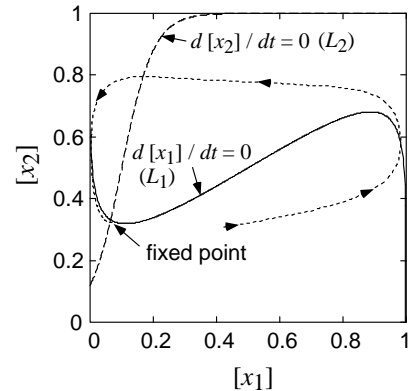

(b) excitation
Figure 10: Nullclines and trajectories of the proposed analog cell in (a) oscillatory mode and (b) excitatory mode.

local connections between cells are used to diffuse the values of the system variables, i.e., diffusion of the chemical species.

We now introduce an analog cell that is qualitatively equivalent to the Oregonator. We define the dynamics of a cell as

$$
\begin{aligned}
\frac{d\left[x_{1}\right]}{d t} & =\frac{1}{\tau}\left(-\left[x_{1}\right]+f\left(\left[x_{1}\right]-\left[x_{2}\right], \beta_{1}\right)\right), \\
\frac{d\left[x_{2}\right]}{d t} & =-\left[x_{2}\right]+f\left(\left[x_{1}\right]-\theta, \beta_{2}\right)
\end{aligned}
$$

where $f(\cdot)$ represents a sigmoid function defined by

$$
f(x, \beta)=\frac{1+\tanh \beta x}{2} .
$$

The cell dynamics are designed so that the shape of nullclines and flows $\left(\left[\dot{x_{1}}\right],\left[\dot{x_{2}}\right]\right)$ are qualitatively equivalent to that of the Oregonator. The cubic nullcline ( $l_{1}$ in Fig. 1$)$ is approximated by a nullcline of Eq. (15) as

$$
\left[x_{2}\right]=\left[x_{1}\right]-\beta_{1}^{-1} \tanh ^{-1}\left(2\left[x_{1}\right]-1\right),\left(\equiv L_{1}\right)(18)
$$

while the linear nullcline ( $l_{2}$ in Fig. 1$)$ is approximated by a nullcline of Eq. (16) as

$$
\left[x_{2}\right]=f\left(\left[x_{1}\right]-\theta, \beta_{2}\right) . \quad\left(\equiv L_{2}\right)
$$

An analog cell, whose dynamics are described by Eqs. (15) and (16), is very suitable for analog VLSI implementation because the sigmoid function can easily be implemented on the VLSIs by using differential-pair circuits.

The proposed cell exhibits qualitatively equivalent behavior to the Oregonator, as shown in Fig. 10. The values of the parameters are $\tau^{-1}=10, \beta_{1}=5$ and $\beta_{2}=10$. When $\theta=0.5$, the fixed point exists on a nulllcline [Eq. (18)] where $d\left[x_{2}\right] / d\left[x_{1}\right]>0$, and the system exhibits limitcycle oscillations [Fig. 10(a)]. On the other hand, the system exhibits excitatory behavior [Fig. 10(b)] when the fixed

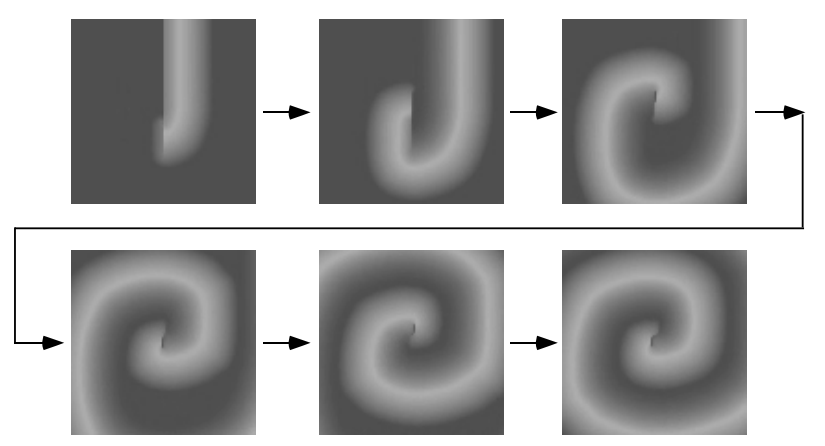

(a) $D_{u}=5 \times 10^{-4}, \theta=0.14$

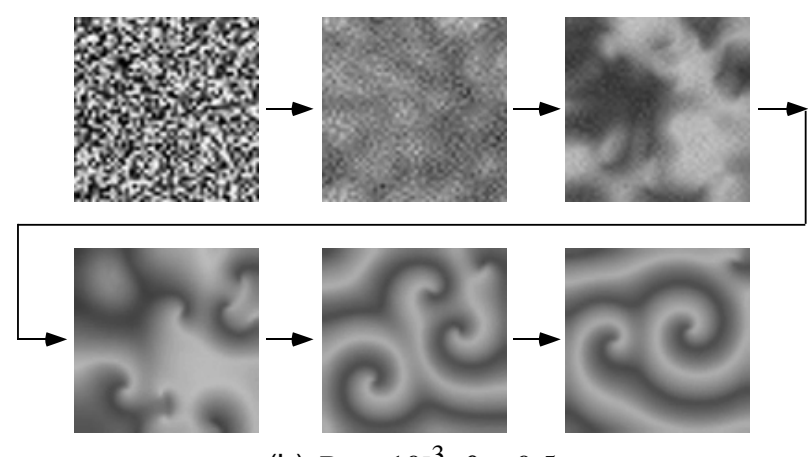

(b) $D_{u}=10^{-3}, \theta=0.5$

Figure 11: Numerical results of a RD system using the ana$\log$ cell in (a) excitatory mode and (b) oscillatory mode.

point exists on a nulllcline (19) where $d\left[x_{2}\right] / d\left[x_{1}\right]<0$ [Fig. 10(b)].

Now, let us introduce the cell dynamics into the basic RD model for the purpose of constructing a 2D CA system. The dynamics of the CA are obtained by substituting the right terms of Eqs. (15) and (16) for the nonlinear reactive functions $f_{u}(\cdot)$ and $f_{v}(\cdot)$ in Eqs. (10) and (11), and with the transformation of the system variables $\left(\left[x_{1}\right] \rightarrow\left[u_{i, j}\right]\right.$ and $\left.\left[x_{2}\right] \rightarrow\left[v_{i, j}\right]\right)$. The resultant dynamics of a cell are

$$
\begin{gathered}
\frac{d\left[u_{i, j}\right]}{d t}=\frac{1}{\tau}\left(-\left[u_{i, j}\right]+f\left(\left[u_{i, j}\right]-\left[v_{i, j}\right], \beta_{1}\right)\right)+g_{i, j}^{u}, \\
\frac{d\left[v_{i, j}\right]}{d t}=-\left[v_{i, j}\right]+f\left(\left[u_{i, j}\right]-\theta, \beta_{2}\right)+g_{i, j}^{v}
\end{gathered}
$$

where $g_{i, j}^{u}$ and $g_{i, j}^{v}$ represent external inputs to the cell (interactions between a cell and its neighboring cells) defined in $\S 2$.

Figure 11 shows spatiotemporal activities of the analog CA with $50 \times 50$ cells $\left(\beta_{1}=5, \beta_{2}=10, h=0.01\right.$ and $D_{v}=0$ ) where the values of $v_{i, j}$ are represented in grayscale $\left(v_{i, j}=0\right.$ : black, $v_{i, j}=1$ : white). The Neumann boundary condition was applied at the side of the square reaction-space. When $\tau^{-1}=10^{2}$ and $\theta=0.14$ at which 


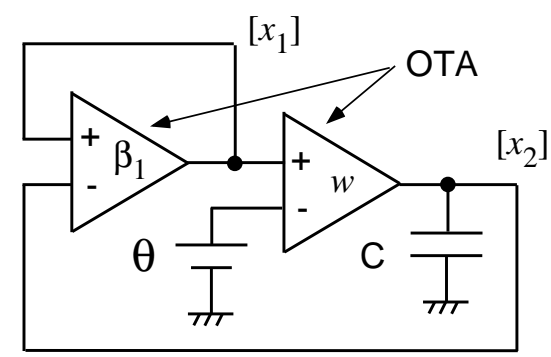

Figure 12: An analog cell circuit consisting of single capacitor and two operational-transconductance amplifiers (OTAs).

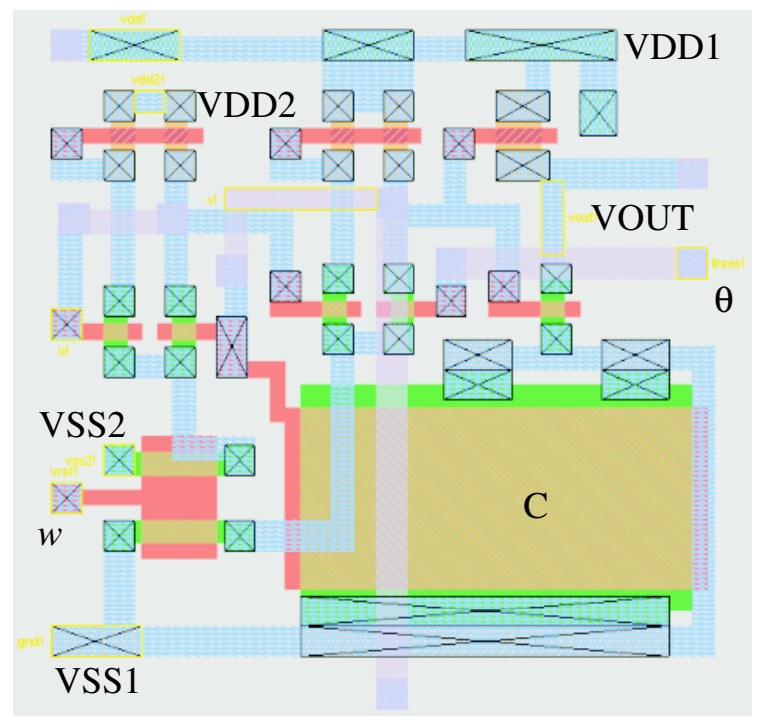

Figure 13: Layout of the analog cell designed with $1.5-\mu \mathrm{m}$ CMOS process (cell size: $70 \times 70 \mu \mathrm{m}^{2}$ ).

the cell exhibits excitatory behavior, the 2D CA system produced spiral patterns [Fig. 11(a)], as is observed in the basic RD system with the Oregonators (Fig. 3). In the simulation, the diffusion coefficient $D_{u}$ was set at $5 \times 10^{-4}$, and initial states of the cells are set at the same states as in Fig. 3. The results indicate that the proposed analog CA is qualitatively equivalent to the basic RD system with the Oregonators since the excitatory property of the analog cells is inherently the same as that of the Oregonator.

Figure 11(b) shows the dynamic behaviors of the analog CA with $D_{u}=10^{-3}$ and $\theta=0.5$, at which the cell exhibits oscillatory behavior. Initial values of the cells were randomly chosen as $\left[u_{i, j}\right]=\operatorname{RAND}[0,1]$ and $\left[v_{i, j}\right]=$ RAND $[0,1]$. The CA produced 2D phase-lagged stable synchronous patterns called modelock, due to weak coupling between the neighboring cells. When $D_{u}>10^{-3}$, all cells exhibit synchronous oscillation. In other words, no spatial pattern was produced.
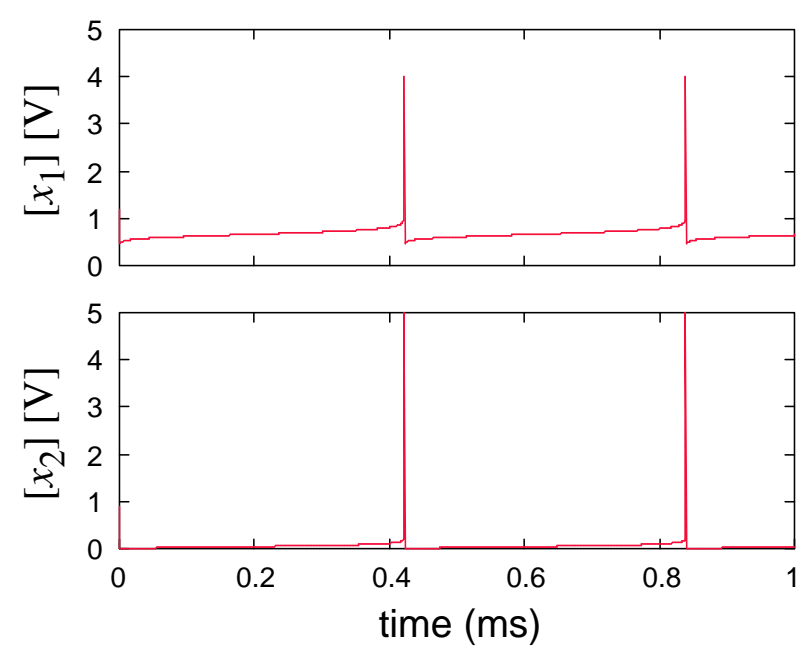

Figure 14: SPICE results of an extracted cell circuit from Fig. 13.

An analog circuit of the proposed cell and its device layout are shown in Figs. 12 and 13, respectively. The circuit consists of single capacitor and two operationaltransconductance amplifiers (OTAs), which implies that the circuit can easily be implemented on silicon VLSIs using conventional CMOS technology. The circuit can be obtained by qualitative approximation of Eqs. (15) and (16).

When the rate constant of Eq. (15) is much larger than that of Eq. (16), the differential term of Eq. (15) can be neglected $(\tau \ll 1)$, as explained in $\S 2$. On the other hand, Eq. (16) with $\beta_{2} \rightarrow \infty$ forces the values of variable $\left[x_{2}\right]$ to be 0 when $\left[x_{1}\right] \leq \theta$, and while $\left[x_{2}\right] \rightarrow 1$ when $\left[x_{1}\right]>\theta$. If the variable $\left[x_{2}\right]$ is forced to have the value within the range $[0: 1]$, the temporal difference in Eq. (16) can approximately be represented by binary values. Consequently, we obtain a new dynamical equation from Eqs. (15) and (16) as

$$
\begin{aligned}
{\left[x_{1}\right] } & =f\left(\left[x_{1}\right]-\left[x_{2}\right], \beta_{1}\right) \\
\frac{d\left[x_{2}\right]}{d t} & = \begin{cases}w & \left(\text { if }\left[x_{1}\right]>\theta\right) \\
-w & (\text { else })\end{cases}
\end{aligned}
$$

where $w$ represents a positive and small constant. In Fig. 12, an OTA labeled as $\beta_{1}$ serves as the function of Eq. (22), while a capacitor $C$ and the other OTA, receiving voltage $\theta$, produce the dynamics for Eq. (23). The positive constant $w$ is implemented in the OTA (labeled as $w$ ) where $w$ corresponds to the source current of a differential pair. The output current of the OTA $(w)$ becomes 0 when the voltage of the output node $\left[x_{2}\right]$ is equal to the supply voltage (VDD or VSS). The value of $\left[x_{2}\right]$ is thus restricted within the range [VDD:VSS].

Figure 14 shows SPICE results of an extracted circuit from the device layout shown in Fig. 13. A MOSIS $1.5-\mu \mathrm{m}$ 
CMOS technology file with a transistor model of BSIM3 (level 8) was used to evaluate the circuit with an actual device layout. The supply voltages of the OTA of $\beta_{1}$ were set at $\mathrm{VDD}=4 \mathrm{~V}$ and VSS $=0.5 \mathrm{~V}$, while that of the other OTA $(w)$ were set at VDD $=5 \mathrm{~V}$ and VSS $=$ GND. The threshold $\theta$ was set at $2.5 \mathrm{~V}$ so that the circuit would exhibit oscillatory behavior. In the device layout shown in Fig. 13, the capacitor $C$ was implemented by a MOS capacitor (lowerright rectangle in Fig. 13). As expected, the circuit exhibited the qualitatively same behavior as the Oregonator; i.e., stiff nonlinear oscillations. The size of the analog cell was $70 \times$ $70 \mu \mathrm{m}^{2}$.

\section{Summary}

We introduced silicon devices that imitate autocatalytic and dissipative phenomena of reaction-diffusion (RD) systems. Numerical simulations showed that the RD device can successfully produce concentric and spiral waves in the same way as natural RD systems. These results encourage us to develop new applications based on natural RD phenomena using hardware RD devices.

The proposed devices and circuits are useful not only for the hardware RD system but also for constructing modern neuro-chips. The excitatory and oscillatory behaviors of the RD device and circuit are very similar to actual neurons that produce sequences of identically shaped pulses in time, called spikes. Recently, Fukai showed that an inhibitory network of spiking neurons achieves robust and efficient neural competition on the basis of a novel timing mechanism of neural activity [19]. A network with such a timing mechanism may provide an appropriate platform for the development of analog VLSI circuits and could overcome the problems of analog devices, namely their lack of precision and reproducibility.

\section{References}

[1] G. Nicolis and I. Prigogine, Self-organization in Nonequilibrium Systems - From Dissipative Structures to Order through Fluctuations. John Wiley \& Sons, Inc., 1977.

[2] R. J. Field and M. Burger, Oscillations and travelling waves in chemical systems. John Wiley \& Sons, Inc., 1985.

[3] H. Tanaka and A. Hasegawa: Electronics Letters 38 (2002) 186.

[4] R. Kapral and K. Showalter Eds, Chemical waves and patterns. Kluwer Academic Publishers, Boston, 1985.
[5] L. Kuhnert, K. I. Agladze, and V. I. Krinsky, "Image processing using light-sensitive chemical waves," $\mathrm{Na}$ ture, Vol. 337, pp. 244-245, 1989.

[6] M. Hiratsuka, T. Aoki, and T. Higuchi, "Pattern formation in reaction-diffusion enzyme transistor circuits," IEICE Trans. Fundamentals, Vol. E82-A, No. 9, pp. 1809-1817, 1999.

[7] O. Steinbock, Á. Tóth, and K. Showalter, "Navigating complex labyrinths: Optimal paths from chemical waves," Science, Vol. 267, pp. 868-871, 1995.

[8] I. Motoike and K. Yoshikawa, "Information operations with an excitable field," Phys. Rev. E, Vol. 59, pp. 53545360, 1999.

[9] T. Asai, Y. Nishimiya, and Y. Amemiya: IEICE Trans. Fundamentals E85-A (2002) 2093.

[10] T. Asai, Y. Nishimiya, and Y.Amemiya: Proceedings. Int. Semiconductor Device Research Symp (2001), 141-4.

[11] Y. Nishimiya, T. Asai, and Y.Amemiya: Ext. Abst. 2001 Int. Conf. on Solid State Devices and Materials (2001), 404-5.

[12] H. Kato, T. Asai, and Y. Amemiya: Proceedings. 5th Int. Conf. on Cognitive and Neural Systems (2001), P41.

[13] Á. Tóth, V. Gáspár, and K. Showalter: J. Phys. Chem 98 (1994) 522.

[14] T. Toffoli and N. Margolus: Cellular Automata Machines MIT Press, Cambridge, 1987.

[15] A. Adamatzky: Identification of Cellular Automata Taylor and Francis, London, Bristol, 1994.

[16] K. Preston, Jr. and M. J.B. Duff: Modern Cellular Automata: Theory and Applications (Plenum Press, New York, 1984) p. 17.

[17] T. Asai, T. Sunayama, Y. Amemiya, and M. Ikebe: Jpn. J. Appl. Phys. 40 (2001) 2585.

[18] T. Sunayama, M. Ikebe, T. Asai and Y. Amemiya: Jpn. J. Appl. Phys. 39 (2000) 2278.

[19] T. Fukai: Biol. Cybern. 75 (1996) 453. 